\title{
Plantations de bois d'œuvre en zone équatoriale africaine : cas de l'arboretum de l'Enef de Mbalmayo au sud du Cameroun
}

Pierre-André OWONA Ndongo ${ }^{1}$

Régis PeltieR ${ }^{2}$

Ibrahim LINJOUOM ${ }^{3}$

Dominique LouPPE ${ }^{2}$

Georges SMEKTALA 4

Vincent BELIGNÉ ${ }^{5}$

Raphaël NJouKaM ${ }^{1}$

Bernard TIECHE ${ }^{3}$

Lucie Temgoua ${ }^{6}$

1 Irad, Cra-Ekona

PMB 25, Buéa

Cameroun

2 Cirad-Es

Campus international de Baillarguet

TA C-36/D

34398 Montpellier cedex 5

France

3 Enef

BP 69, Mbalmayo

Cameroun

${ }^{4}$ AgroParisTech-Engref

BP 7353

34086 Montpellier cedex 4

France

5 Scac

Ambassade de France

BP 1616, Yaoundé

Cameroun

6 Fasa

Université de Dschang

BP 96, Dschang

Cameroun

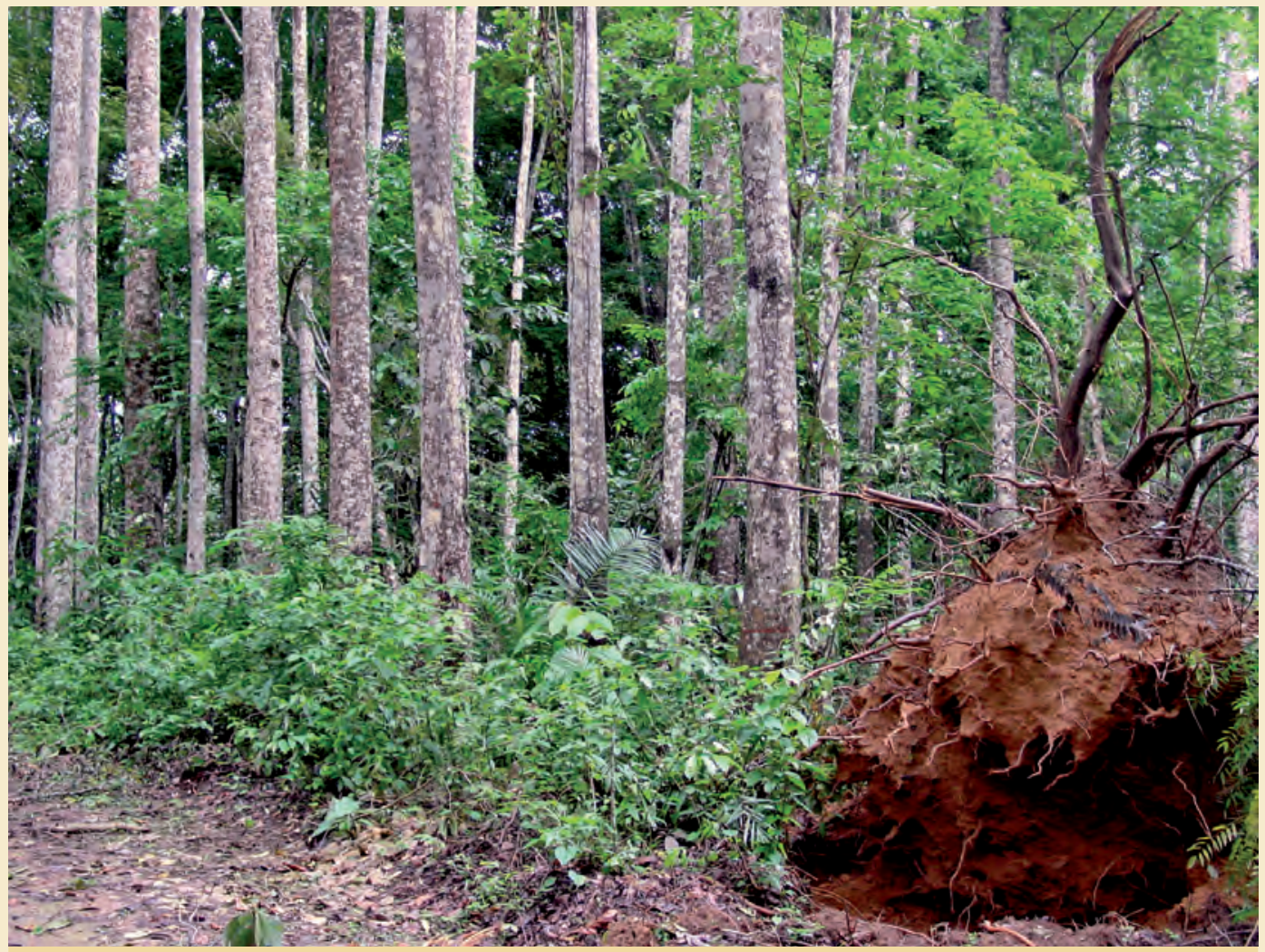

Chablis dans la parcelle de framiré.

Photo Régis Peltier. 


\section{RÉSUMÉ}

\section{PLANTATIONS DE BOIS D' GUVRE EN ZONE ÉQUATORIALE AFRICAINE : CAS DE L'ARBORETUM DE L'ENEF DE MBALMAYO AU SUD DU CAMEROUN}

Aux abords de l'École forestière de Mbalmayo au Cameroun, un arboretum a été installé à partir de 1956. Il a été suivi et protégé par plusieurs générations d'enseignants avec l'appui de diverses agences de coopération. À l'occasion du cinquantième anniversaire de sa création, une étude y a été menée sur quinze parcelles afin de mesurer leurs principaux paramètres dendrométriques et de pouvoir estimer leur productivité en bois d'œuvre. Les peuplements de bété, Mansonia altissima, de framiré, Terminalia ivorensis, et de wengé, Milletia laurentii, présentent une bonne production ligneuse qui augure de belles perspectives pour le reboisement. Les parcelles, au départ monospécifiques, abritent à présent une régénération naturelle variée d'espèces ligneuses et herbacées. Ces résultats permettent de justifier une reprise des plantations de bois d'œuvre en zone de forêt dense humide d'Afrique centrale. Les grandes plantations industrielles, qui ne sont plus aujourd'hui prioritaires, cèdent utilement la place à de petites plantations individuelles ou à des boisements collectifs ou communaux plus étendus. Le Programme sectoriel Forêt et Environnement financé par un mécanisme de remise de la dette doit y contribuer.

Mots clés : plantation forestière, arboretum, enseignement, Cameroun, productivité.

\section{ABSTRACT}

\section{PLANTATIONS FOR CONSTRUCTION TIMBER IN EQUATORIAL AFRICA: THE MBALMAYO FORESTRY SCHOOL ARBORETUM IN SOUTHERN CAMEROON}

The arboretum established in 1956 around the Mbalmayo Forestry School in Cameroon has been monitored and cared for by several generations of teaching staff, with the support of various cooperation agencies. Marking the fiftieth anniversary of its creation, a study was carried out in fifteen parcels in order to measure the main dendrometric parameters and assess their productivity for construction timber. High timber productivity in several species, Bété (Mansonia altissima), Framiré (Terminalia ivorensis) and Wengé (Milletia laurentii), offers good prospects for reforestation. Though initially planted as single-species stands, a variety of woody and herbaceous species is now regenerating naturally in the different parcels. These results justify renewed planting of construction timber species in the dense humid forest zones of central Africa. Large industrial plantations, which are no longer a priority today, are being usefully replaced by small individual plantations or more extensive community or village plantations. The Sectoral Forests and Environment Programme, which is financed under a debt cancellation scheme, should contribute to this.

Keywords: forest plantation, arboretum, teaching, Cameroon, productivity.

\section{RESUMEN}

\section{PLANTACIONES DE MADERA DE CONSTRUCCIÓN EN UNA ZONA ECUATORIAL AFRICANA: EL CASO DEL ARBORETO DE LA ENEF DE MBALMAYO EN EL SUR DE CAMERÚN}

En 1956 se estableció un arboreto en los alrededores de la Escuela Forestal de Mbalmayo, en Camerún. De su seguimiento y protección se han encargado varias generaciones de docentes con el respaldo de diferentes agencias de cooperación. Con ocasión del quincuagésimo aniversario de su creación, se llevó a cabo un estudio en quince parcelas para medir sus principales parámetros dendrométricos y así poder estimar su productividad en madera de construcción. Los rodales de bété, Mansonia altissima, framiré, Terminalia ivorensis, y wengé, Milletia laurentii, presentan una buena producción de madera que augura buenas perspectivas para la reforestación. Las parcelas, en un principio monoespecíficas, albergan actualmente una regeneración natural variada de especies leñosas y herbáceas. Estos resultados permiten justificar la reanudación de las plantaciones de madera en zonas de bosque húmedo y denso de África Central. Las grandes plantaciones industriales, que ya no son prioritarias, dejan ahora paso a pequeñas plantaciones individuales o a repoblaciones colectivas o comunales más extensas y útiles. El Programa Sectorial Forêt et Environnement, financiado por un mecanismo de condonación de la deuda, debe contribuir a ello.

Palabras clave: plantacion forestal, arboreto, enseñanza, Camerún, productividad. 


\section{Introduction}

\section{Cadre de l'étude}

Les premières plantations forestières de bois d'œuvre furent réalisées au Sud-Cameroun par les forestiers allemands, il y a plus d'un siècle. Après 1918, ils furent relayés par leurs collègues français, puis par les services camerounais et par de nombreux projets. Après tout ce temps, on peut encore s'interroger sur la faisabilité sociale et économique de telles opérations (FRRCirad-Coillte, 2002). En effet, sur les milliers d'hectares plantés au Sud-Cameroun, seule une infime partie a eu une croissance correcte et a été respectée par les défrichements agricoles. Au cours des dix dernières années, pratiquement aucun boisement nouveau n'y a été mis en place. En dehors de quelques coupes dans les plantations d'okoumé de Kribi et dans quelques plantations de teck, la presque totalité du bois exploité dans cette région provient toujours des forêts naturelles. Le même constat peut être fait dans les pays voisins (Congo, Gabon, République centrafricaine). Or la demande nationale, régionale et internationale ne fait qu'augmenter, alors que le potentiel ligneux exploitable des massifs forestiers naturels facilement accessibles va en décroissant.

Deux des freins à l'émergence de plantations dans cette zone, soulignés par l'étude Frr, sont le manque de connaissances sur la productivité en bois d'œuvre exploitable - il existe beaucoup de données sur la croissance juvénile en diamètre et en hauteur mais peu sur la productivité des plantations en volume de fût d'arbres adultes - et la sous-estimation des freins socio-économiques liés à la plantation.

C'est pourquoi, à l'occasion des 50 ans d'existence de l'arboretum de Mbalmayo et en vue de mieux le valoriser pour la professionnalisation des étudiants de l'École nationale des eaux et forêts de Mbalmayo (ENEF, 2003) qui en a la gestion (PELTIER,

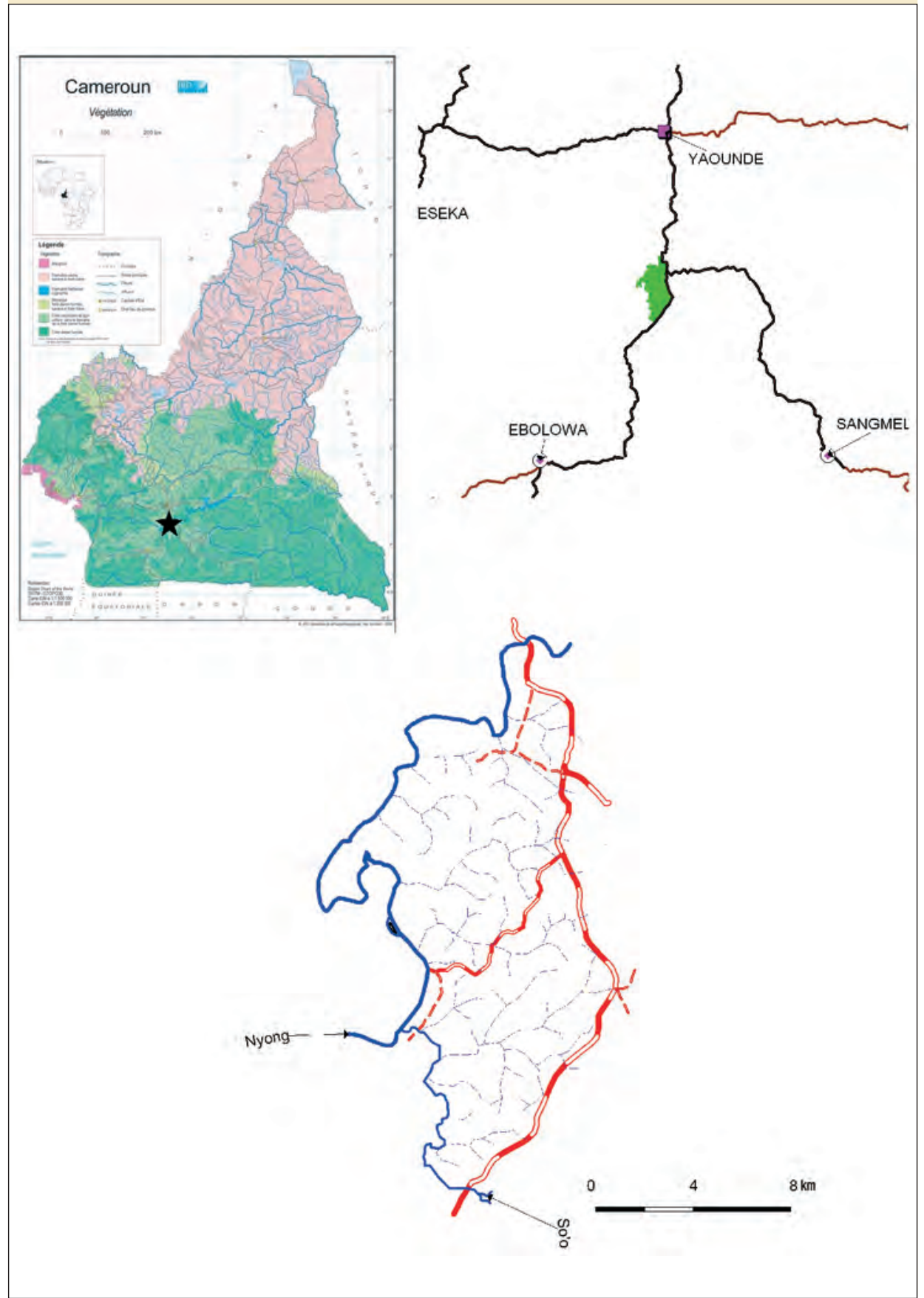

Figure 1.

Localisation de la forêt classée de Mbalmayo.

Carte P.-A. Owona Ndongo.

Njoukam, 2006), il fut décidé de réaliser la présente étude suivant un inventaire des quinze meilleurs placeaux pour estimer leur productivité. Ce recul dans le temps constitue l'intérêt majeur de cet article.
Par ailleurs, une enquête auprès des populations riveraines a été menée pour évaluer leur perception et leur impact vis-à-vis des peuplements de l'arboretum (encadré 1). 


\section{Présentation succincte de l'arboretum}

La forêt a été classée à l'époque où la densité de la population était faible, sur des territoires de culture et de chasse. L'arrêté colonial n² 269 du 29 juillet 1947 portant création de la réserve forestière de Mbalmayo stipule, en son article 2, que : "Les plantations de cacaoyers entretenues et en bon état seront maintenues, et de nouvelles plantations ne se feront qu'en remplacement des anciennes. » L'article 3 précise que : « Les autochtones, possesseurs coutumiers du sol, sont autorisés à établir leurs cultures vivrières à l'intérieur du périmètre classé à la condition de respecter les arbres qui seront indiqués par le Service des Eaux et Forêts. » Cela a engendré une « logique du défrichement et de la course à la terre " pour se constituer un " patrimoine foncier ». Puis, progressivement, les paysans ont associé des arbres fruitiers tels que manguiers, avocatiers, safoutiers (Dacryodes edulis) et colatiers aux cultures vivrières. Ces arbres servent de marqueurs fonciers et de titre traditionnel de propriété.

La forêt classée de Mbalmayo est une forêt périurbaine. Le développement de la ville et l'accroissement de sa population ont augmenté la pression sur la réserve pour ce qui est du bois de chauffe et des terres agricoles. Lors des entretiens, un paysan a dit à ce sujet : "Nous, autochtones de ce village, ne sommes pas une menace pour la réserve, parce que nous connaissons les arbres utiles qu'il faut préserver lors de la mise en place de nos cultures [...] par contre la réserve est envahie par les populations de la ville qui viennent y installer leurs champs, scier et récolter le bois. »

La pression sur la ressource ligneuse s'exerce également sur l'arboretum en termes de prélèvement de bois de chauffage et d'écorces. Il en est résulté la disparition du placeau de Guibourtia tessmannii, le ralentissement de croissance et la malformation de plusieurs espèces (Baillonella toxisperma, Piptadeniastrum africanum, Alstonia boonei, etc.) qui sont très prisées en pharmacopée. Les pièges de braconnage sont nombreux en lisière de l'arboretum : « Les animaux qui sont dans cette forêt consomment nos cultures et notre volaille, nous ne pouvons faire autrement... » Cette chasse illégale concerne des rongeurs (aulacodes, porcs-épics, hérissons...), des herbivores (céphalophes, biches...) et des petits carnivores (renards, civettes...). Ces gibiers sont très prisés par la population et sont parfois commercialisés en bordure de route.

Finalement, malgré des revendications foncières récurrentes, les populations admettent qu'elles tirent des avantages de la proximité de ces plantations où elles effectuent diverses récoltes de bois ou d'autres produits provenant des arbres ou de la chasse. Elles pensent aussi que la mise en valeur de ces plantations pourrait constituer une opportunité d'emploi.

Les agents de l'administration, pour leur part, estiment urgent que la tenure foncière et l'accès aux ressources forestières soient reprécisés pour sauver cette forêt envahie et pillée à la fois par les populations riveraines, qui disent jouir de leurs droits traditionnels, et par les populations de Mbalmayo, pour lesquelles la forêt constitue un lieu « vacant », ouvert à tous pour la pratique de l'agriculture et la coupe illégale du bois.

L’arboretum, placé sous la gestion de l'Enef, se trouve à une cinquantaine de kilomètres de Yaoundé, en bordure de la ville de Mbalmayo. II occupe une superficie de 5,8 ha au sein de la forêt domaniale de Mbalmayo, classée en 1947, et qui couvre 9700 ha (OWONA NDONGo et al., 2008).

Le climat y est guinéen bimodal avec une pluviosité annuelle moyenne de $1600 \mathrm{~mm}$, répartie en deux saisons des pluies (de mars à juin et de septembre à novembre). La température annuelle moyenne est de $23^{\circ} \mathrm{C}$ et l'hygrométrie moyenne de $78 \%$. L'arboretum est situé à une altitude de $640 \mathrm{~m}$ sur sol à sesquioxydes ferrallitiques désaturés jaunes dont la roche mère est un schiste (Fодном, 1983). La végétation originelle est une forêt de transition entre la forêt semi-décidue et la forêt dense sempervirente (AUBRÉVILLE, 1956).

L'arboretum a été créé à partir de 1956, après une coupe à blanc de la forêt. Une cinquantaine d'espèces y ont été plantées. L'unité parcellaire est un carré de $25 \mathrm{~m}$ de côté planté d'une seule espèce. L'écartement à la plantation est de $2,5 \mathrm{~m}$ en tous sens, soit une densité de 1600 tiges à l'hectare. Deux parcelles contiguës sont séparées par une ligne non plantée (NGASSA, 1989). Alors que la plupart des plantations de la forêt domaniale de Mbalmayo ont été dévastées par les défrichements agricoles, l'exploitation illégale et le feu, l'arboretum a été protégé par chaque génération d'enseignants, avec divers appuis et financements extérieurs, dont celui de la coopération soviétique. 


\section{Méthodologie}

Pour faire le choix des parcelles et des essences à prendre en compte et à inventorier, ont été exclues les parcelles présentant une mortalité trop importante ou dans lesquelles une régénération abondante d'espèces pionnières n'aurait pas permis à l'espèce de développer tout son potentiel et, par ailleurs, ont été retenues les essences présentant une valeur économique certaine et faisant l'objet d'un commerce régulier (tableau I).

Pour évaluer le comportement des essences de bois d'œuvre plantées, deux étapes ont été requises : - D’une part, la connaissance de l'état des plantations par l'appréciation des paramètres suivants: la composition et la structure dans un plan vertical (strates), la nature de la régénération naturelle, le taux de survie de l'essence plantée, les traitements sylvicoles appliqués à la parcelle et l'état sanitaire des arbres.

- D’autre part, la mesure des arbres sur pied pour construire des tarifs de cubage spécifiques afin d'estimer le volume de bois d'œuvre.

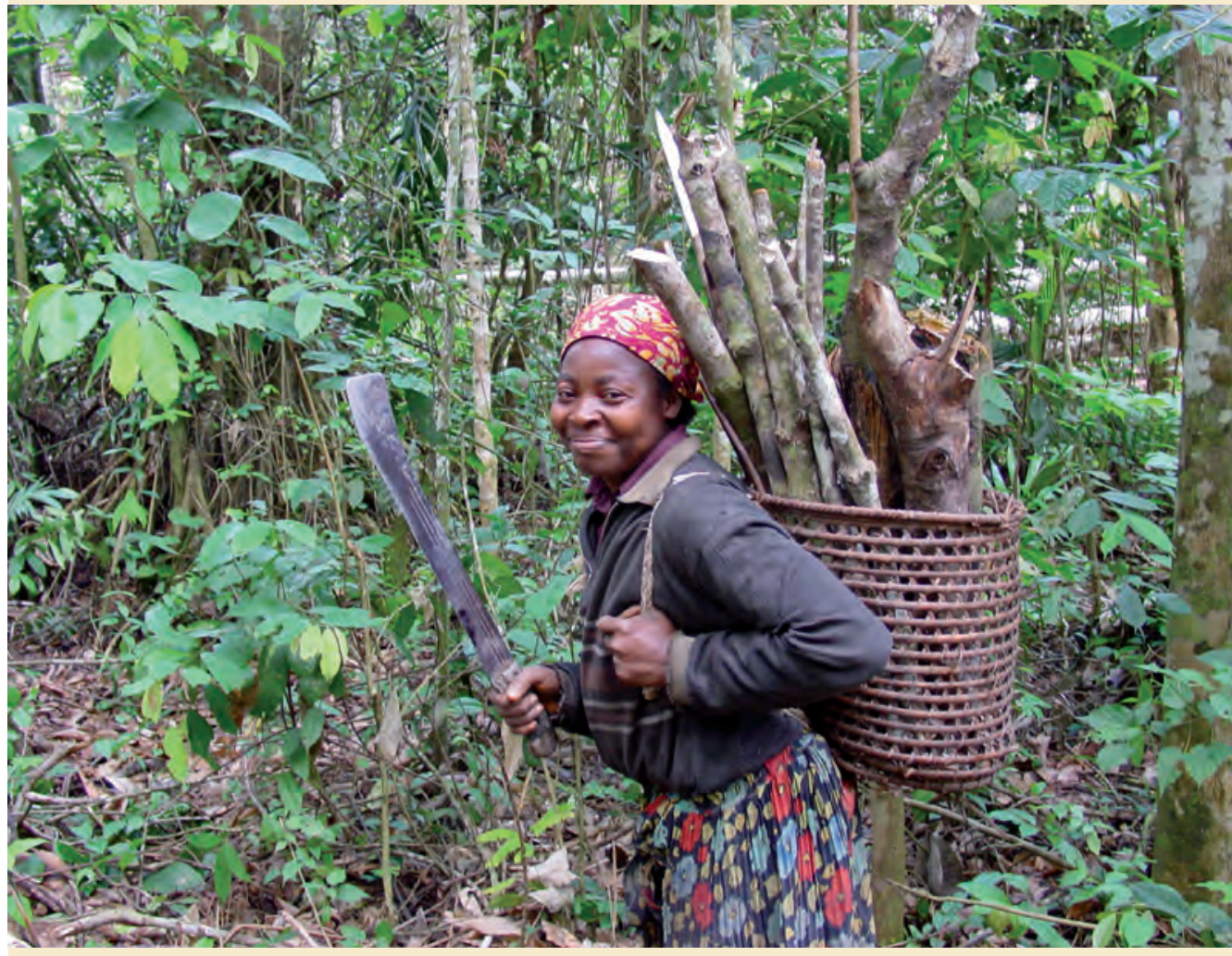

Profitant d'une tolérance tacite, une villageoise d'Oyak récolte du bois de feu dans l'arboretum.

Photo Régis Peltier.
Tableau I.

Les essences forestières concernées.

Nom scientifique

Aucoumea klaineana

Diospyros crassiflora

Distemonanthus benthamianus

Entandrophragma candollei

Entandrophragma cylindricum

Eribroma oblonga

Erythroxylum mannii

Khaya ivorensis

Lophira alata

Mansonia altissima

Millettia laurentii

Pinus kesiya

Sterculia rhinopetala

Terminalia ivorensis

Triplochiton scleroxylon
Famille

Burséracées

Ébénacées

Caesalpiniacées

Méliacées

Méliacées

Sterculiacées

Erythroxylacées

Méliacées

Ochnacées

Sterculiacées

Fabacées

Pinacées

Sterculiacées

Combrétacées

Sterculiacées
Compte tenu de la faible superficie des parcelles $(0,0625 \mathrm{ha})$, un inventaire en plein donnant le nombre de tiges par hectare $(\mathrm{N})$ et le taux de survie (S \%) a été réalisé (il faut noter que la mortalité des arbres est due à la concurrence intraspécifique, il n'y a eu ni éclaircie, ni exploitation frauduleuse, ni défaut d'entretien). La circonférence de chaque arbre a été mesurée au mètre ruban à 1,30 $\mathrm{m}$ au-dessus du niveau du sol ou $0,30 \mathrm{~cm}$ au-dessus des contreforts, pour les espèces qui en développent, ce qui a permis de calculer la surface terrière $(G)$ et le diamètre de l'arbre de surface terrière moyenne $\left(d_{g}\right)$.

La hauteur totale des arbres a été mesurée au relascope de Bitterlich. La hauteur dominante $\left(\mathrm{H}_{0}\right)$ du peuplement a été estimée par la moyenne arithmétique des hauteurs totales des quatre plus gros arbres 


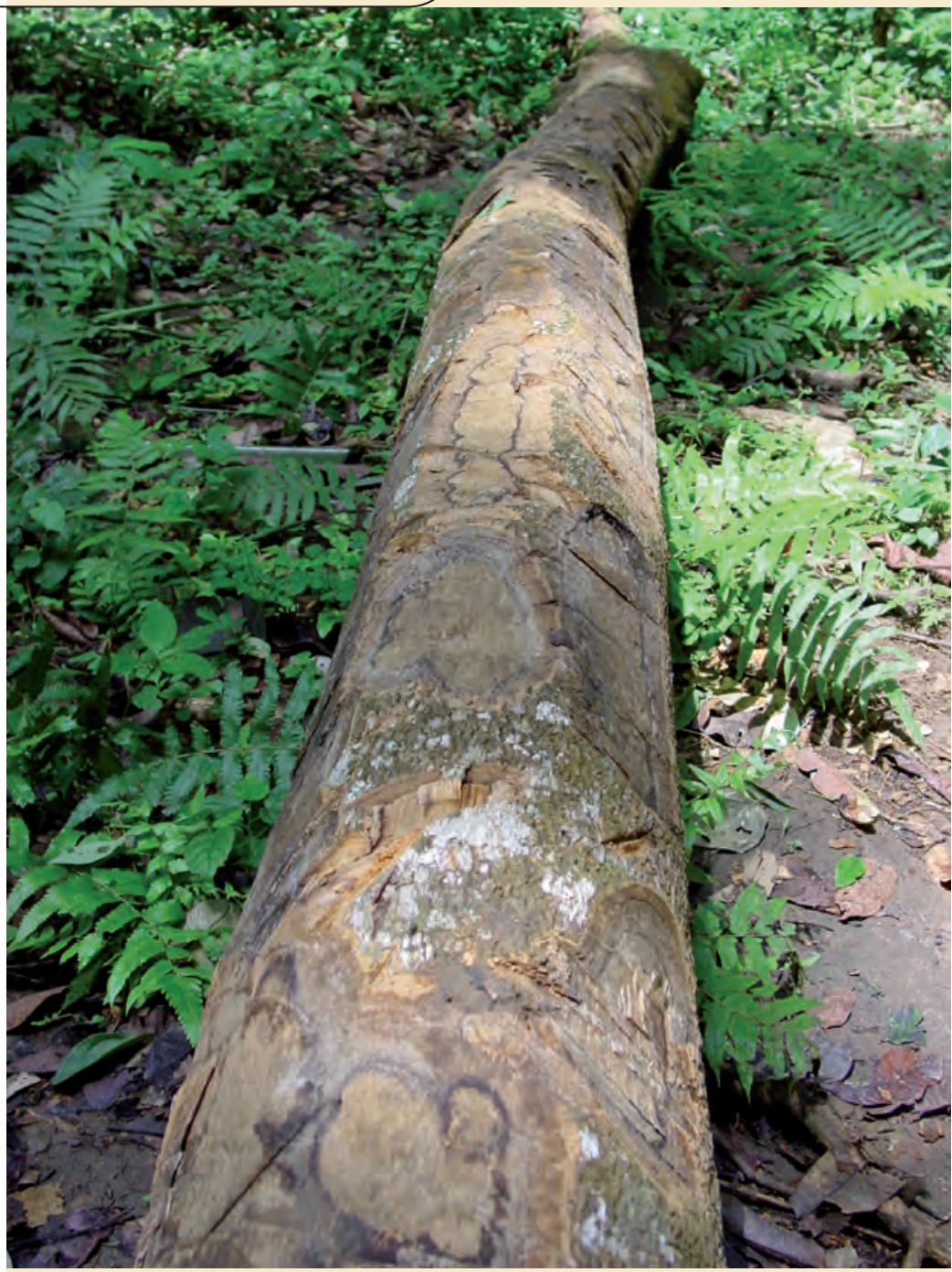

Les habitants de la ville de Mbalmayo récoltent les écorces à usages pharmacologiques sur plusieurs espèces plantées dans l'arboretum, comme ce chablis de Alstonia boonei.

Photo Régis Peltier.

du placeau (au lieu des cinq arbres recommandés par PARDE et BOU CHON, 1988).

Pour établir les tarifs de cubage, quinze arbres par espèce ont été cubés sur pied, au relascope de Bitterlich, en veillant à ce que les différentes classes de diamètre soient représentées.

Le volume du fût $\left(\mathrm{V}_{\mathrm{f}}\right)$ ou volume commercial de chaque arbre, comprenant la bille de pied et les surbilles éventuelles, est la somme des volumes des tronçons le composant, calculé au moyen de la formule de Smalian. Le volume du houppier n'a pas été estimé, faute de présenter une grande valeur commerciale pour le bois d'œuvre.
Deux équations de cubage de la forme: $V=b_{0}+b_{1} c+b_{2} c^{2}$ et $V=b_{0} c^{b}$ ont été établies pour chacune des quinze essences retenues, avec $\mathrm{V}$ (volume en $\mathrm{m}^{3}$ ), $\mathrm{C}$ (circonférence en $m$ ) et $b_{0}, b_{1}$ et $b_{2}$ (constante et coefficients de régression). Remarque : ces tarifs ne sont applicables que dans les conditions bien particulières de l'arboretum, c'est pourquoi leur intérêt est limité aux calculs intermédiaires et ne sont pas présentés dans la partie "Résultats" (OwonA Ndongo, 2006). Le choix de l'équation la plus fiable a été effectué après examen de la dispersion des résidus autour de la courbe de régression et comparaison des coefficients de détermination $\mathrm{R}^{2}$.

\section{Résultats}

\section{Description des parcelles}

Les placeaux ont été matérialisés en 1956 et lors des deux ou trois années suivantes. Les arbres étaient donc âgés de 50 ans au moment des mesures. Les plantations n'ont reçu aucun soin cultural, en dehors des dégagements des premières années.

En localisant spatialement tous les arbres vivants, trois structures ont été observées : une distribution aléatoire (eyong, movingui, sapelli... ; figure $2 a$ ), une distribution régulière (azobé, wengé... ; figure 2b) et une distribution irrégulière, en lignes ou en agrégats (framiré, bété, kosipo, landa, lotofa... ; figure $2 \mathrm{c}$ ).

Du point de vue spécifique, les peuplements sont généralement hétérogènes car plus ou moins envahis par la régénération des essences des parcelles voisines, sauf les peuplements d'ébène et de wengé restés purs car leur couvert est très dense. Néanmoins, dans le mélange, l'essence plantée constitue généralement la strate dominante, à l'exception du sapelli et de l'eyong, qui se cantonnent dans le sous-étage. Le sapelli est surcimé par Alstonia boonei, Erythroxylum mannii et Terminalia ivorensis. L'eyong est dominé par Terminalia superba, Lovoa trichilioides, Pterygota macrocarpa, Pycnanthus angolensis et Canarium schweinfurthii. Ces deux essences ont vu leur croissance fortement ralentie par la concurrence. Elles n'ont donc pas pu exprimer tout leur potentiel productif.

\section{État des peuplements}

À l'exception de quelques cas isolés d'attaques d'insectes et de pestes, l'état sanitaire des peuplements est satisfaisant. Cependant, tous les sapellis (Entandrophragma cylindricum) présentent de nombreuses crosses ou fourches dues à des piqûres d'insectes, probablement des attaques de borers, Hypsipyla robusta, lors du jeune âge, ce qui 


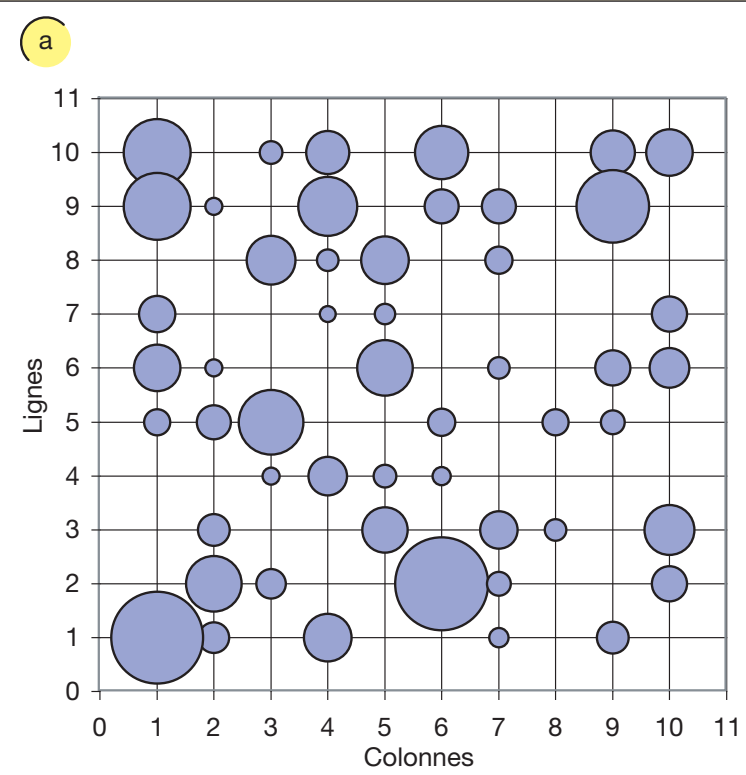

OPieds d'ayous

Figure 2a.

Parcelle de Triplochiton scleroxylon : distribution aléatoire (la taille des cercles est proportionnelle à la surface terrière individuelle).

\section{Figure $\mathbf{2 b}$.}

Diospyros crassiflora :

distribution régulière liée à une très faible mortalité.

Figure 2c.

Terminalia ivorensis :

distribution irrégulière, les arbres sont essentiellement cantonnés en bordure de parcelle, le long de la piste. (b

productivité des plantations / LE POINT SUR..

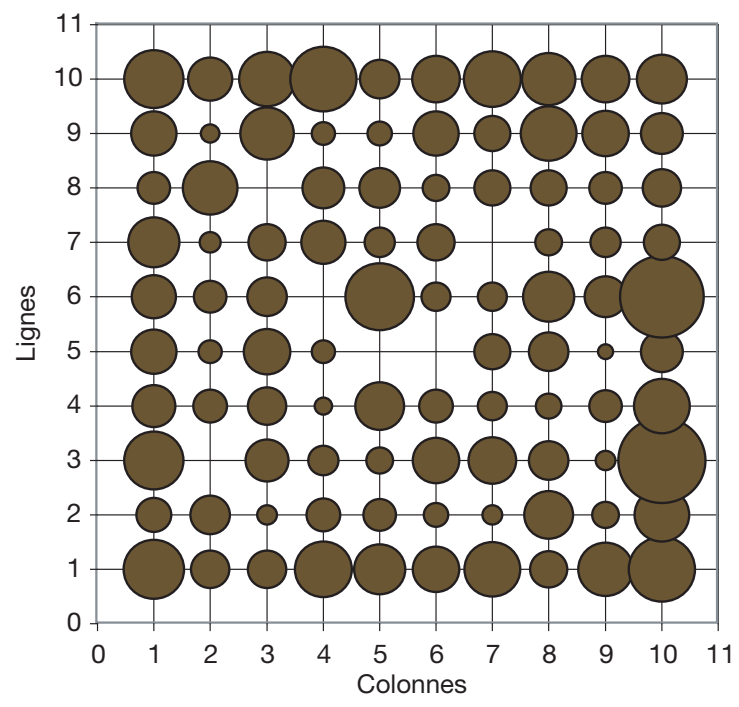

Pieds d'ébène<smiles></smiles>

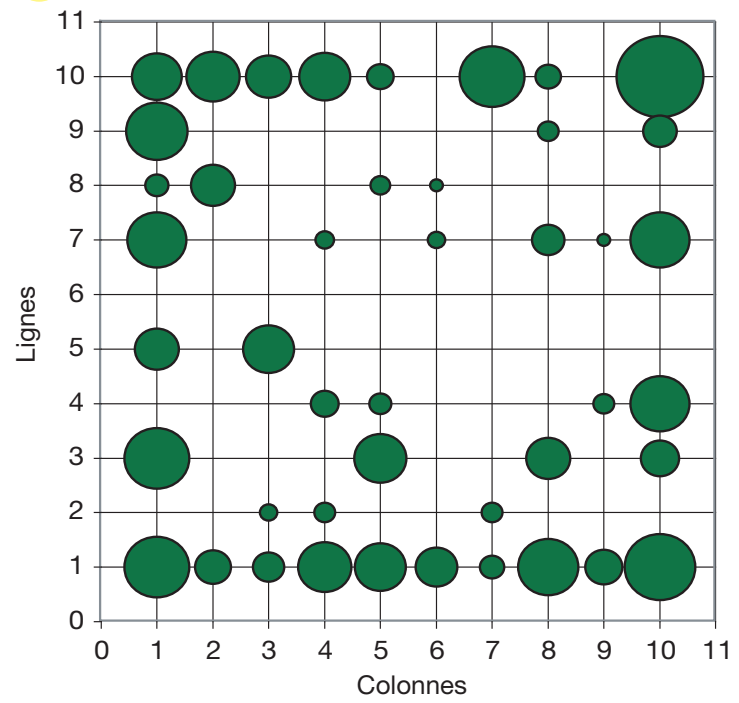

Pieds de framiré expliquerait leur retard initial de croissance et leur surcimage par des arbres issus de la régénération naturelle d'autres espèces. D'éventuelles atta ques de borers pourraient aussi être à l'origine du très faible taux de survie de l'acajou (Khaya ivorensis).

L'élagage naturel s'opère assez bien dans l'ensemble des parcelles, sauf pour Pinus kesiya qui aurait nécessité un élagage artificiel. Tous les peuplements sont également denses et comportent majoritairement des arbres de petit diamètre. Ils ont donc souffert du manque de syl- viculture et ne se sont éclaircis que par une sélection naturelle consécutive à une trop forte concurrence. Une densité trop élevée donne aussi des arbres très élancés sensibles au moindre coup de vent et aux chablis. La parcelle de framiré (Terminalia ivorensis) a été victime d'un chablis important en 2006, avec la chute simultanée de plusieurs arbres, ce qui a permis de les cuber plus précisément. Concernant la forme des troncs, on peut noter que les okoumés sont tordus et peu cylindriques.

\section{Caractéristiques dendrométriques des peuplements}

Le tableau II présente les données dendrométriques de chacune des parcelles. Ces données sont à prendre avec précaution car les parcelles sont de petite taille et entourées d'un chemin. Les surfaces théoriques utiles des parcelles vont donc jusqu'au centre de ce chemin et couvrent $900 \mathrm{~m}^{2}(30 \times 30 \mathrm{~m})$ et non $625 \mathrm{~m}^{2}(25 \times 25 \mathrm{~m})$. Cette surface théorique est celle qui a été utilisée 


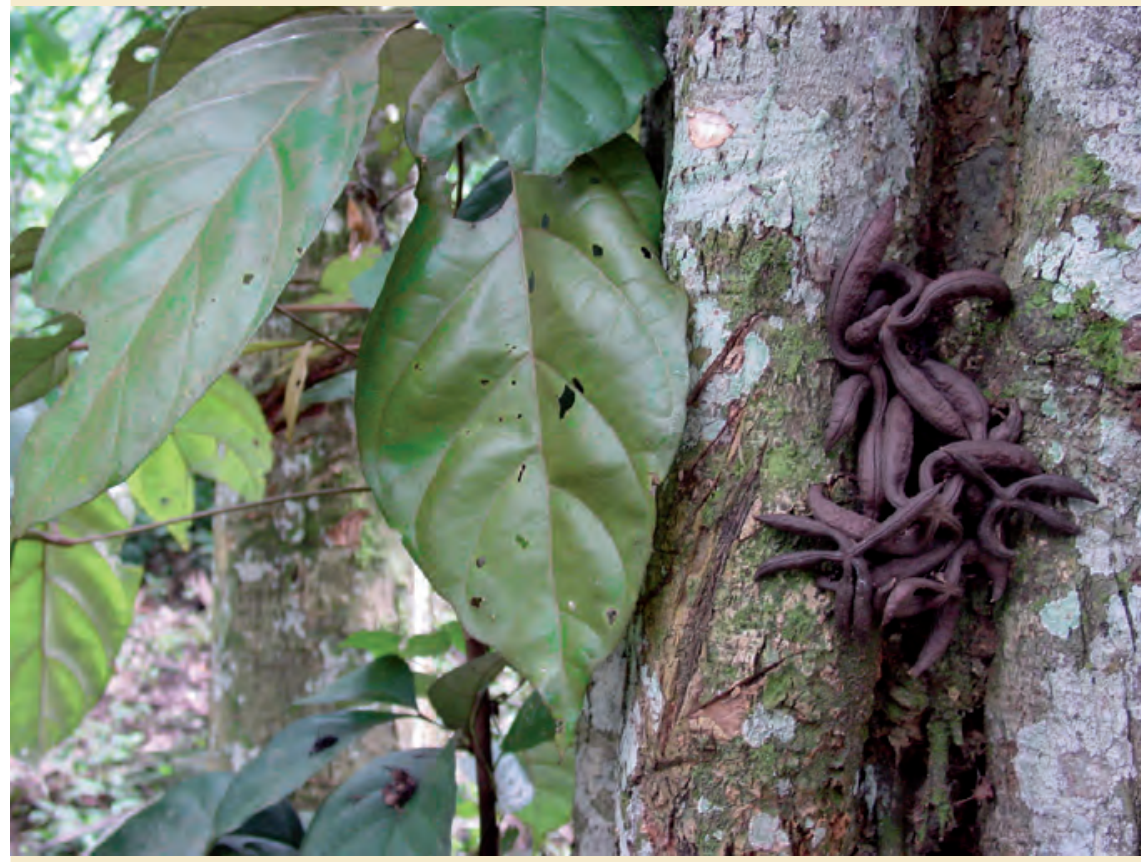

Fruitier sauvage planté, comme marqueur foncier, sur une parcelle proche de l'arboretum.

Photo Régis Peltier. pour les estimations. Elle est exacte dans le cas de parcelles entourées de parcelles portant un peuplement fermé avec un volume sur pied identique. Elle ne l'est plus si une ou plusieurs parcelles voisines sont vides ou portent des arbres peu développés. Dans ce cas, les branches et les racines de l'espèce plantée envahissent peu ou prou l'espace de la parcelle voisine. Pour éviter les biais, il aurait fallu prendre les projections des houppiers de la ligne de bordure comme limite de parcelle, ce qui aurait donné une estimation assez fiable de la surface réellement couverte par les arbres de la parcelle étudiée. Faute de temps, il n'a pas été possible de le faire.

La projection à l'hectare de la densité des arbres vivants varie selon l'espèce, de 250 pour le bété à plus de 1000 pour l'ébène. Les taux de survie sont particulièrement élevés pour l'ébène (94\%), l'azobé (88\%) et le wengé $(81 \%)$. De ces trois espèces, seul le wengé montre une bonne croissance en diamètre malgré

Tableau II.

Paramètres dendrométrique des parcelles.

Espèce
Aucoumea klaineana
Diospyros crassiflora
Distemonanthus benthamianus
Entandrophragma candollei
Entandrophragma cylindricum
Eribroma oblonga
Erythroxylon mannii
Khaya ivorensis
Lophira alata
Mansonia altissima
Millettia laurentii
Pinus kesiya
Sterculia rhinopetala
Terminalia ivorensis
Triplochiton scleroxylon

\begin{tabular}{|c|c|c|c|c|c|c|c|c|}
\hline S (\%) & N/ha & $\mathrm{H}_{\text {dom }}$ & $D_{g}$ & G & V/ha & AAM & $\mathrm{D}_{\max }$ & $A A D_{\text {max }}$ \\
\hline 45 & 500 & 29 & 45 & 80 & 840 & 16,8 & 73 & 1,4 \\
\hline 94 & 1040 & 24 & 22 & 40 & 320 & 6,4 & 40 & 0,8 \\
\hline 53 & 590 & 26 & 26 & 30 & 270 & 5,4 & 52 & 1,0 \\
\hline 47 & 520 & 20 & 24 & 24 & 180 & 3,6 & 64 & 1,3 \\
\hline 50 & 550 & 11 & 17 & 13 & 68 & 1,3 & 33 & 0,7 \\
\hline 50 & 550 & 16 & 13,5 & 7,7 & 40 & 0,8 & 24 & 0,5 \\
\hline 47 & 520 & 28 & 33 & 44 & 510 & 10,2 & 55 & 1,1 \\
\hline 29 & 320 & 22 & 42 & 44 & 380 & 7,6 & 69 & 1,4 \\
\hline 88 & 980 & 28 & 22 & 39 & 260 & 5,2 & 45 & 0,9 \\
\hline 23 & 250 & 31 & 35 & 25 & 290 & 5,8 & 50 & 1,0 \\
\hline 81 & 900 & 23 & 35 & 90 & 810 & 16,3 & 83 & 1,7 \\
\hline 42 & 460 & 33 & 47 & 82 & 890 & 18,0 & 71 & 1,4 \\
\hline 43 & 470 & 25 & 28 & 29 & 250 & 5,1 & 50 & 1,0 \\
\hline 44 & 480 & 36 & 52 & 105 & 1690 & 33,8 & 79 & 1,6 \\
\hline 51 & 560 & 30 & 35 & 54 & 570 & 11,4 & 69 & 1,4 \\
\hline
\end{tabular}

$\mathrm{S}$ (\%) (taux réel de survie à 50 ans); $\mathrm{N} /$ ha (densité à l'hectare); $\mathrm{H}_{\text {dom }}$ (hauteur dominante en $\mathrm{m}$ ); $\mathrm{D}_{\mathrm{g}}$ (diamètre de l'arbre de surface terrière moyenne en $\mathrm{cm}$ ); $\mathrm{G}$ (surface terrière en $\mathrm{m}^{2} / \mathrm{ha}$ ); $\mathrm{V} / \mathrm{ha}$ (volume par hectare des fûts sur écorce en $\mathrm{m}^{3} / \mathrm{ha}$ ) ; AAM (accroissement annuel moyen en $\mathrm{m}^{3} / \mathrm{ha} / \mathrm{an}$ ) ; $\mathrm{D}_{\max }$ (moyenne quadratique du diamètre des trois plus gros sujets de la parcelle en $\mathrm{cm}$ ); $A A D_{\text {max }}$ (accroissement annuel moyen en diamètre des trois plus gros sujets de la parcelle en $\mathrm{cm}$ ). Toutes les valeurs ont été arrondies à l'unité ou à la dizaine inférieure, sauf pour les AA où on a conservé un chiffre significatif après la virgule. 


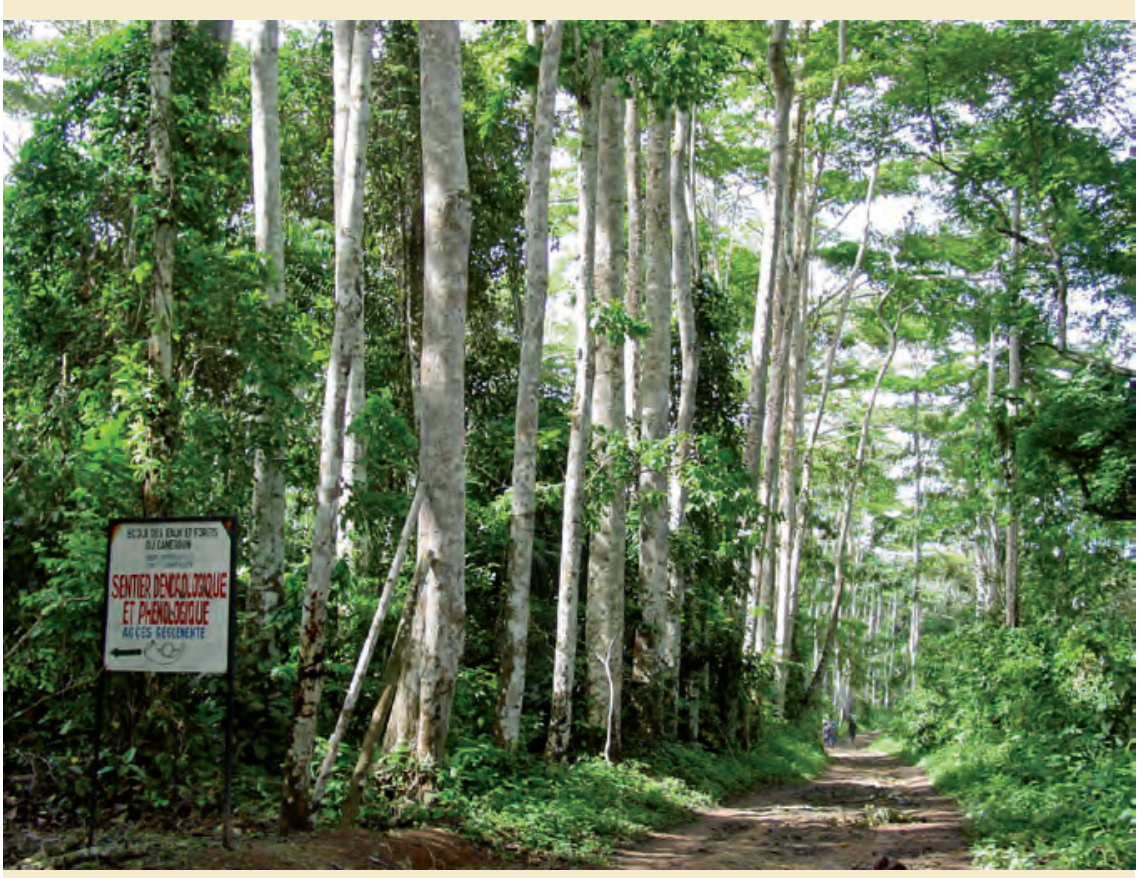

Une belle ligne de dibétou ( Lovoa trichilioides Harms) forme la limite nord de l'arboretum. Photo Régis Peltier.

Tableau III.

Accroissements diamétriques annuels (en $\mathrm{mm}$ ) des essences étudiées.

\begin{tabular}{|c|c|c|c|}
\hline \multirow[b]{2}{*}{ Essence } & \multicolumn{3}{|c|}{ Accroissement diamétrique (mm/an) } \\
\hline & Le plus élevé & Moyenne & Le moins bon \\
\hline Ayous & 15 & 6,6 & 2 \\
\hline Azobé & 11 & 3,9 & 2 \\
\hline Bété & 10 & 9,3 & 2 \\
\hline Ébène & 8 & 3,8 & 1 \\
\hline Eyong & 5 & 2,7 & 1 \\
\hline Framiré & 20 & 7,1 & 3 \\
\hline Kosipo & 14 & 4,4 & 1 \\
\hline Landa & 12 & 5,0 & 2 \\
\hline Lotofa & 10 & 4,4 & 2 \\
\hline Movingui & 11 & 4,9 & 1 \\
\hline Acajou & 16 & 8,0 & 1 \\
\hline Okoumé & 15 & 8,3 & 3 \\
\hline P. kesiya & 14 & 9,4 & 4 \\
\hline Sapelli & 7 & 3,0 & 1 \\
\hline Wengé & 18 & 6,1 & 1 \\
\hline
\end{tabular}

la forte densité des arbres. Les taux de survie du bété ( $23 \%$ ) et de l'acajou (29\%) sont les plus faibles. Les autres essences ont des taux de survie de 40 à $50 \%$. Le taux de survie n'est qu'un critère indicatif non représentatif du peuplement car, dans la parcelle de framiré, par exemple, de nombreux plants dominés sont restés petits avec une production insignifiante. Pour cette essence, le diamètre de l'arbre le plus gros est de $102 \mathrm{~cm}$ (soit un accroissement de $2 \mathrm{~cm}$ par an), alors que celui des deux plus petits n'est que de $17 \mathrm{~cm}$. En effet, à un écartement initial de 2,5 $\mathrm{m}$ et sans intervention, l'effet de lisière joue beaucoup en faveur des individus qui ont survécu.

Quatre essences (okoumé, wengé, Pinus kesiya et framiré) présentent, selon la méthode de calcul appliquée, une surface terrière supérieure à $60 \mathrm{~m}^{2} / \mathrm{ha}$, qui est le maximum communément admis. Au Gabon, l'okoumé en plantations avec 1400 tiges à l'hectare peut atteindre $30 \mathrm{~m}^{2}$ de surface terrière et une production annuelle moyenne de $25 \mathrm{~m}^{3} /$ ha à 10 ans (LEROY-DeVAL, 1976). L'accroissement en diamètre des okoumés dominants peut se maintenir à $1,25 \mathrm{~cm} /$ an jusqu'à l'âge de 40 ans (NASI, 1997). Les données obtenues à Mbalmayo pour les arbres pris individuellement sont du même ordre de grandeur. Pour ces quatre espèces, il est possible d'estimer qu'elles ont atteint leur surface terrière maximale.

Le tableau III présente les accroissements en diamètre extrêmes et moyens des quinze essences.

De grands écarts de croissance sont constatés entre les arbres d'une même essence au sein d'un même peuplement. La croissance la plus rapide a été observée chez les sujets bénéficiant de l'effet de bordure dans toutes les parcelles, ce qui mène à penser que, si des éclaircies avaient été pratiquées dans l'arboretum de Mbalmayo, la croissance des arbres aurait été bien meilleure et plus homogène. 


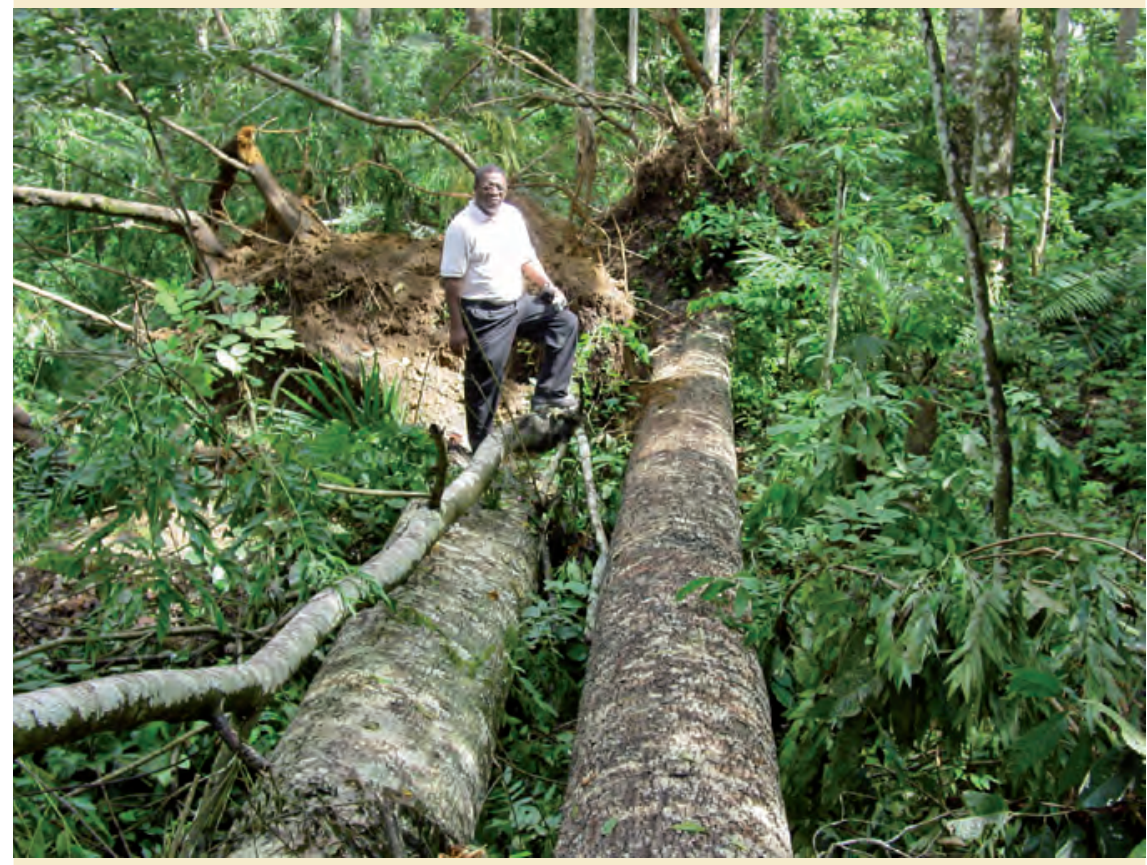

Chablis 2006 dans la parcelle de framiré. Noter que les arbres de cinquante ans avaient déjà atteint un diamètre exploitable. Photo Régis Peltier.

Le Mémento du forestier (MINISTÈrE DE LA COOPÉRATION, 1989) préconise pour le framiré une sylviculture dynamique qui permet de récolter 70 arbres de $60 \mathrm{~cm}$ de diamètre entre 35 et 40 ans, pour une surface terrière de $20 \mathrm{~m}^{2} / \mathrm{ha}$.
L'accroissement annuel moyen en diamètre selon cette sylviculture est identique à celui des trois plus gros arbres de la parcelle inventoriée à Mbalmayo. Ainsi la croissance, dans l'arboretum, des trois plus gros arbres de chaque espèce pourrait-elle représenter la croissance moyenne d'un peuplement ayant bénéficié d'une sylviculture dynamique ramenant le nombre d'arbres à 70 par hectare en moins de 50 ans.

En posant cette hypothèse sylvicole, on obtient une estimation basse de la productivité (tableau IV), car les récoltes intermédiaires ne sont pas connues.

En dehors de l'okoumé, dont la forme n'est pas bonne, et de Pinus kesiya, très branchu, on constate que Khaya ivorensis, Millettia laurentii, Terminalia ivorensis et Triplochiton scleroxylon auraient, à 50 ans, un diamètre de l'arbre de surface terrière moyenne égal ou supérieur à $70 \mathrm{~cm}$ et un accroissement annuel moyen égal ou supérieur à $5 \mathrm{~m}^{3} / \mathrm{ha}$. Une telle production de bois d'œuvre préjuge de bonnes perspectives économiques de rentabilité. Toutefois, le calcul économique n'a pas pu être fait, faute de données sur le prix du bois sur pied au Cameroun et dans la sous-région.

Tableau IV.

Estimation du volume de bois d'œuvre sur pied de peuplements âgés de $\mathbf{5 0}$ ans et ramenés à une densité de $\mathbf{7 0}$ tiges par hectare par une sylviculture dynamique.

\begin{tabular}{|l|c|r|r|r|r|}
\hline & AAM en D & $\mathrm{D}_{50}$ & $\mathrm{G}_{50}$ & $\mathrm{~V}_{50}$ & AAM en V \\
\hline Aucoumea klaineana & 1,4 & 70 & 26,9 & 334 & 6,7 \\
\hline Diospyros crassiflora & 0,8 & 40 & 8,8 & 100 & 2,0 \\
\hline Distemonanthus benthamianus & 1,0 & 50 & 13,7 & 120 & 2,4 \\
\hline Entandrophragma candollei & 1,3 & 65 & 23,2 & 186 & 3,7 \\
\hline Erythroxylon mannii & 1,1 & 55 & 16,6 & 200 & 4,0 \\
\hline Khaya ivorensis & 1,4 & 70 & 26,9 & 269 & 5,4 \\
\hline Mansonia altissima & 1,0 & 50 & 13,7 & 172 & 3,5 \\
\hline Millettia laurentii & 1,7 & 85 & 39,7 & 414 & 8,3 \\
\hline Pinus kesiya & 1,4 & 70 & 26,9 & 339 & 6,8 \\
\hline Sterculia rhinopetala & 1,0 & 50 & 13,7 & 130 & 2,6 \\
\hline Terminalia ivorensis & 1,6 & 80 & 35,5 & 713 & 14,3 \\
\hline Triplochiton scleroxylon & 1,4 & 70 & 26,9 & 253 & 5,1
\end{tabular}

$A A M$ en $D$ (accroissement annuel moyen sur le diamètre en $\mathrm{cm}$ ) ; $D_{50}$ (diamètre de l'arbre de surface terrière moyenne à 50 ans en $\mathrm{cm}$ ); $\mathrm{G}_{50}$ et $\mathrm{V}_{50}$ (surface terrière en $\mathrm{m}^{2} /$ ha et volume sur pied en $\mathrm{m}^{3} /$ ha à 50 ans) ; AAM en $V$ (accroissement annuel moyen du peuplement sur pied en $\mathrm{m}^{3} / \mathrm{ha} / \mathrm{an}$ ). 


\section{Discussion}

\section{Accroissement diamétrique moyen}

Les résultats obtenus sur le kosipo (4,3 mm/an), le sapelli (2,9 mm/an), le lotofa (4,4 mm/an), l'okoumé $(8,3 \mathrm{~mm} / \mathrm{an})$ et Pinus kesiya $(9,4 \mathrm{~mm} / \mathrm{an})$ vont dans le sens des autres recherches qui ont été menées sur la croissance des arbres en forêt dense africaine. Au Ghana, les accroissements diamétriques sont de l'ordre de 4 à $5 \mathrm{~mm} / \mathrm{an}$ pour le kosipo (ADLER, 1989). En Côte d'Ivoire, l'accroissement diamétrique est de 2,8 mm/an pour le sapelli à Mopri (DURRIEU DE MADRon et al., 1997, 1998). Au Gabon, est avancé un accroissement diamétrique de l'okoumé en peuplements purs de 5 à 15 mm/an (BRUNCK et al., 1990). $\mathrm{Au}$ Cameroun, est obtenu un accroissement diamétrique de $4 \mathrm{~mm} / \mathrm{an}$ pour le lotofa (DURRIEU DE MADRON et al., 1998). Dans l'Ouest-Cameroun, a été mesuré un accroissement diamétrique de 13,1 mm/an, pour une plantation de Pinus kesiya non éclaircie, à 21 ans (NJoukAM, PELtier, 2004). Ainsi, il apparaît que les accroissements obtenus dans le cas de Mbalmayo (tableau IV) sont similaires à ceux enregistrés par d'autres études de référence.

Par ailleurs, le potentiel de croissance individuel du sapelli (7 mm/an) et de l'ayous (15 mm/an) à Mbalmayo entre dans la fourchette des résultats obtenus (JARDIN, 1995) pour les mêmes essences dans différentes forêts semi-décidues au sudest du Cameroun.

\section{Surfaces terrières et volumes}

Les résultats obtenus dans les placeaux de l'arboretum, en termes de surface terrière et de volume, sont difficilement extrapolables à l'hectare car ils sont surestimés (par rapport à un vaste peuplement) en incluant la ligne de bordure et sousestimés en l'éliminant des calculs.
De ce fait, ils doivent n'être considérés qu'avec précaution, d'autant plus que l'âge exact de chaque parcelle n'est connu qu'à deux ou trois ans près. Toutefois, un tel arboretum présente l'avantage de regrouper un certain nombre d'essences sur un même site, ce qui permet de simuler la dynamique comparative d'éventuelles plantations. II représente assez bien le comportement que des essences peuvent avoir en peuplements purs ou en mélange. Les phénomènes de recolonisation des parcelles, par des espèces autres que celles qui ont été installées à l'origine (arbres, arbustes, lianes et herbacées), fournissent des informations importantes pour la gestion des peuplements forestiers et peuvent donner lieu à des études sur la contribution des plantations existantes et des reboisements en vue de la conservation de la biodiversité.

\section{Conclusion}

Cette étude, bénéficiant d'un exceptionnel recul dans le temps, montre qu'un grand nombre d'essences se comportent bien en plantation dans l'arboretum de Mbalmayo. Elles ont une production de bois d'œuvre qui laisse entrevoir une bonne rentabilité économique.

Au contraire, parmi les quinze essences forestières de bois d'œuvre étudiées, le sapelli présente de nombreux nœuds de reprise de croissance dus aux attaques d'insectes et semble peu adapté à ce type de plantation; le sapelli et l'eyong sont les seules essences à présenter une très faible croissance, montrant ainsi une mauvaise adaptation à la zone d'étude.

Cependant, les effectifs par essence, sur lesquels l'étude a été faite, sont trop réduits et ne permettent pas d'établir des tarifs de cubage et de calculer des accroissements précis. Il est donc vivement recommandé que d'autres études soient menées dans ce sens, afin de consolider ces données. Néanmoins, ces premiers

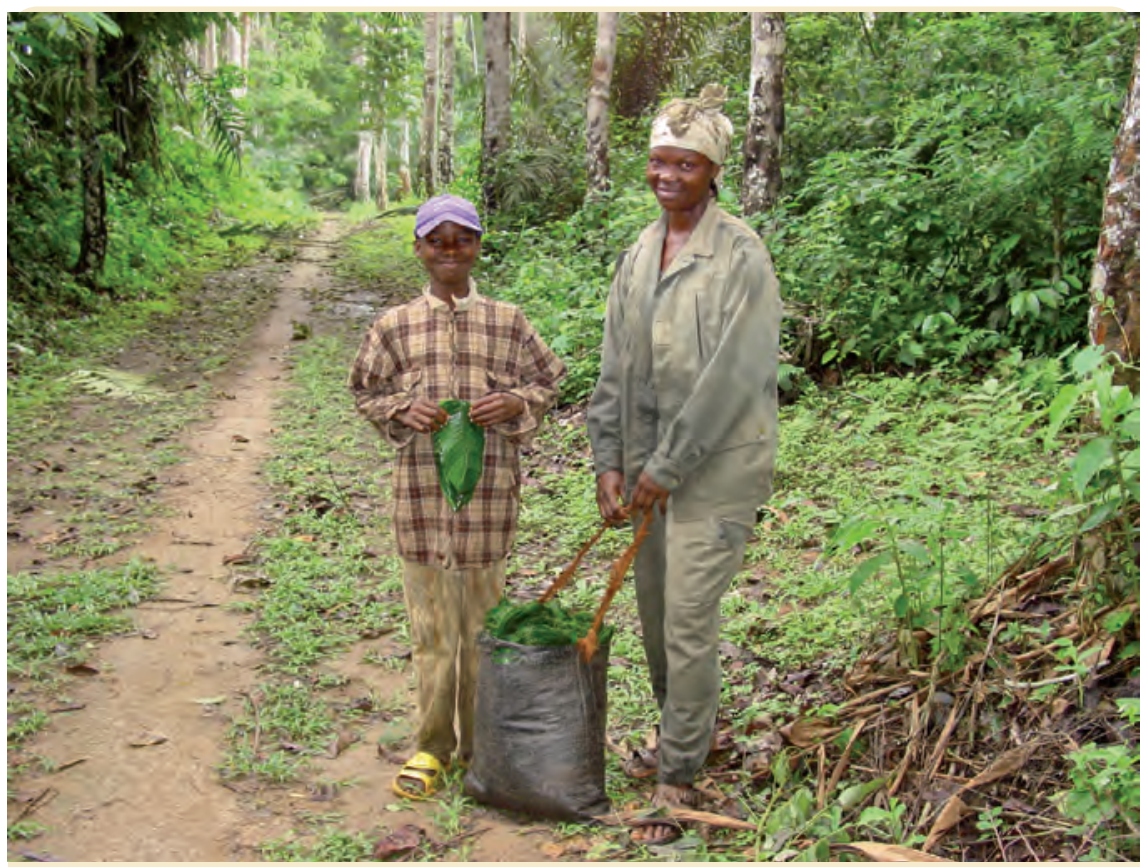

Récolte de feuilles de marantacées (utilisées pour emballer les aliments), herbacées qui poussent à l'ombre des arbres de l'arboretum. Photo Régis Peltier. 


\section{Références}

bibliographiques

résultats peuvent contribuer au calcul de la possibilité des essences étudiées et servir ainsi d'outil d'aide à la décision quant à la mise en œuvre des projets de reboisement.

Enfin, bien que des études précises n'aient pas été réalisées, les observations et les enquêtes auprès des populations montrent que ces parcelles, au départ monospécifiques, abritent une régénération naturelle variée d'espèces ligneuses et herbacées et constituent un abri pour la petite faune. La gestion concertée de ces productions pourrait apporter un appoint nutritionnel et monétaire durable aux populations riveraines.

Tout cela milite en faveur d'une reprise des plantations de bois d’œuvre dans les écosystèmes dégradés de la zone de forêt dense humide d'Afrique centrale. Les grandes plantations industrielles, qui ne sont plus prioritaires aujourd'hui, pourraient utilement faire place à de petites plantations individuelles ou à des boisements collectifs ou communaux plus étendus. Le Programme sectoriel forêt et environnement (Psfe), financé par un mécanisme de remise de dette doit y contribuer, à condition que l'accès au foncier et au crédit soit facilité et que le droit de propriété individuelle sur les plantations soit définitivement clarifié vis-à-vis de l'administration en charge des forêts.
ADLER D., 1989. Natural forest increment, growth and yield. In : Wong J. L. G., Dunn R. M. (éd.). Ghana forest inventory project seminar proceedings, 29-30th March 1989. Accra, Ghana, Overseas Development Administration/Ghana Forestry Department, p. 47-52.

AUBRÉVILLE A., 1956. La flore forestière de la Côte d'ivoire. $2^{\mathrm{e}}$ éd. Nogentsur-Marne, France, Centre technique forestier tropical, tome 1, $371 \mathrm{p}$.

BRUNCK F., GRISON F., MAÎTRE H. F., 1990. L'okoumé (Aucoumea klaineana Pierre), Monographie. Nogentsur-Marne, France, Centre technique forestier tropical, $102 \mathrm{p}$.

DURRIEU DE MADRON L., FAVRICHON V., DUPUY B., BAR HEN A., HOUDE L., MAÎTRE H.-F., 1997. Croissance et productivité en forêt dense humide : bilan des expérimentations dans le dispositif de Mopri, Côte d'Ivoire (1978-1992). Projet Forafri, 75 p.

DURRIEU DE MADRON L., FAVRICHON V., DUPUY B., BAR HEN A., HOUDE L., MAÎTRE H.-F., 1998. Croissance et productivité en forêt dense humide : bilan des expérimentations dans le dispositif d'Irobo, Côte d'Ivoire (1978-1990). Projet Forafri, 68 p.

ENEF, 2003. Dossier technique de l'arboretum de l'École des eaux et forêts de Mbalmayo. Mbalmayo, Cameroun, École nationale des eaux et forêts, $15 \mathrm{p}$.

FOAHOM B., 1983. Note de présentation de l'antenne de Mbalmayo (état d'évancement des recherches). Yaoundé, Cameroun, Institut de recherche agricole, $18 \mathrm{p}$.

FRR-CIRAD-COILLTE, 2002. Étude de faisabilité et de promotion du programme de plantations au Cameroun. Bristol, Royaume-Uni, Frr Ltd, 62 p.

JARDIN J.-L., 1995. Étude de la croissance de l'Ayous, Triplochiton scleroxylon, du Sapelli, Entandrophragma cylindricum, et du Fraké, Terminalia superba. Projet d'aménagement pilote intégré de Dimako, Cameroun, $23 \mathrm{p}$.
LEROY-DEVAL J., 1976. Biologie et sylviculture de l'okoumé. Tome 1 : La sylviculture de l'okoumé. Nogent-surMarne, France, Centre technique forestier tropical, $355 \mathrm{p}$.

MINISTÈRE DE LA COOPÉRATION, 1989. Mémento du forestier. Techniques rurales en Afrique. $3^{\text {e }}$ éd. Paris, France, ministère de la Coopération, $1266 \mathrm{p}$.

NASI R., 1997. Les peuplements d'okoumés au Gabon. Leur dynamique de croissance en zone côtière. Bois et Forêts des Tropiques, 251 (1) : 5-26.

NGASSA M., 1989. Un arboretum pour une formation toujours meilleure. Le Forestier, journal de l'École nationale des eaux et forêts, Mbalmayo, Cameroun, vol. 3 (1989) : 11-13.

NJOUKAM R., PELTIER R., 2004. Première éclaircie par méthodes simplifiées dans une plantation de Pinus kesiya Royle à l'Ouest-Cameroun. Cameroon Journal of Agricultural Science, 1 (1) : 36-41.

OWONA NDONGO P. A., 2006. Évaluation de la potentialité des plantations forestières au Centre-Sud Cameroun : résultats des mesures effectuées dans l'arboretum de Mbalmayo et des enquêtes menées en périphérie de sa réserve. Mémoire de master $3 \mathrm{~A}$, option foresterie rurale et tropicale, Engref, Montpellier, France, 137 p.

OWONA NDONGO P. A., PELTIER R., BELIGNE V., NDJIB G., 2008. Mapping Mbalmayo Forest. Remote sensing helps with the management of cameroonian forest reserve. ITTO Tropical Forest Update, 18 (2) : 9-12.

PARDE J., BOUCHON J., 1988. Dendro-

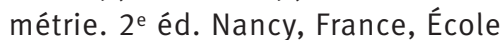
nationale du génie rural, des eaux et forêts, $328 \mathrm{p}$.

PELTIER R., NJOUKAM R., 2006. Plan directeur de formation de l'Enef de Mbalmayo (Cameroun). Minfof-CiradOibt, tomes 1 et 2, 72 p. et 79 p. 